\title{
Nonlinear Disturbance Observer-Based Sliding Mode Control for the PMSM with Matched and Mismatched Disturbances
}

\author{
Yao Fang (D), Huifang Kong (D), and Tiankuo Liu (iD) \\ School of Electrical Engineering and Automation, Hefei University of Technology, Hefei 340000, China \\ Correspondence should be addressed to Huifang Kong; 1227171607@qq.com
}

Received 18 September 2020; Revised 14 November 2020; Accepted 4 December 2020; Published 24 December 2020

Academic Editor: Chaolong Zhang

Copyright ( $) 2020$ Yao Fang et al. This is an open access article distributed under the Creative Commons Attribution License, which permits unrestricted use, distribution, and reproduction in any medium, provided the original work is properly cited.

\begin{abstract}
High-efficiency permanent-magnet synchronous motor (PMSM) is a key technology to improve the driving range of batteries in electric vehicles, while the mismatched disturbance that is caused by external disturbances and parameter perturbation may easily result in speed fluctuations and overshoot of the PMSM, which further deteriorate the performance and efficiency of batteries. To solve the problem, a novel nonlinear disturbance observer-based sliding mode control (NDO-SMC) is proposed. Compared with the traditional SMC method, the NDO-SMC scheme has better disturbance rejection ability in the presence of matched and mismatched uncertainties and disturbances by introducing the estimation value of the nonlinear disturbance observer in the sliding surface. Furthermore, owing to the compensation of the disturbance observer, the switching gain is only required to be greater than the bound of the disturbance estimation error rather than that of the disturbance; thus, the chattering problem is substantially alleviated. A rigorous stability proof of the whole closed-loop system is given in detail using Lyapunov theory by designing an appropriate Lyapunov function. The simulation results demonstrate the feasibility and superiority of the proposed NDO-SMC strategy.
\end{abstract}

\section{Introduction}

Driving range of batteries is a key problem restricting the development of electric vehicles $[1,2]$, permanent-magnet synchronous motor (PMSM), as a high energy-consumption component, whose efficiency becomes the decisive factor for the driving range of batteries [3].

To achieve high-performance control of the PMSM, two current controllers are employed in the FOC mechanism to allow a PMSM to achieve similar torque control performance to a separately excited dc motor, where torque and flux can be controlled separately [4]. On this basis, PID, adaptive control, backstepping control, sliding mode control (SMC), finite-time control, predictive control, and intelligent control algorithms have been applied to improving the control performance from different aspects [5-7].

Among these methods, the SMC method is regarded as the efficient method for possessing the disturbance rejection and robustness properties of PMSM systems [8]. However, chattering phenomenon caused by the discontinuous control term and frequent switching action near the sliding surface is the unavoidable problem, own to the nature of the SMC method. To reduce or even eliminate the chattering effects, a simple and attractive approach is to soften the discontinuous control term by the saturation function or hyperbolic tangent function at the cost of a slight deterioration of control performances [9]. Another alternative is disturbance observer-based sliding mode control (DOBSMC), which provides a promising scheme to handle the chattering phenomenon. Since the disturbances have been precisely estimated by the disturbance observer, design of switching gain only needs to be greater than the disturbance estimation error, rather than the magnitude of the disturbance [10]. In this way, the chattering problem can be alleviated to some extent in the case the nominal performance of the sliding mode control is retained. It is noticed that most existing DOBSMC methods were only applicable for disturbances and uncertainties satisfying the so-called matching condition since traditional SMC is only sensitive to matched disturbance [11]. However, mismatched 
disturbances appeared in different channels from control inputs always exist in the practical control system. In this case, many works $[12,13]$ have been proposed to deal with the control system with mismatched disturbances. Specifically, an integral sliding surface with a high-frequency switching gain is designed, and then integral action in the sliding surface drives the system states to the desired equilibrium in the presence of mismatched uncertainties; it should be pointed out that the integral action also brings some adverse effects into the control system, such as large overshoot and long response time [14]. Another method for handling mismatched uncertainties is focusing on the robustness of the system by Riccati control scheme, which is built on the premise that the mismatch disturbance satisfies $\mathrm{H}_{2}$-norm bound [15].

Inspired by remarkable benefits of the idea proposed in the literature [16], the nonlinear disturbance observer-based sliding mode control (NDO-SMC) method is proposed to deal with the matched and mismatched disturbances in the PMSM control system. The main contributions of the paper are summarized as follows: (1) considering the parameter perturbation and external disturbances of the PMSM, a novel second-order PMSM model with matched and mismatched disturbances is developed; (2) the introduction of disturbance estimation in the sliding surface is to eliminate the influence of the mismatched disturbance on the PMSM system as much as possible. Furthermore, no adverse effect related to the control performance is introduced; (3) due to feedback compensation of matched disturbance, the selection of high-frequency switching gain is only required to be greater than the bound of the disturbance estimation error rather than that of the disturbance, which substantially alleviates the chattering problem.

The rest of the paper is organized as follows: starting from the high-efficiency requirements of batteries in electric vehicles, the basic requirements of permanent-magnet synchronous motor control are given. Considering the external disturbance and parameter perturbation, a secondorder PMSM model with matched and mismatched disturbances is constructed in Section 2. Section 3 gives the NDO-SMC scheme for the PMSM model, and the stability proof of the closed-loop system is also given in detail. The simulations are conducted in Section 4, followed by the conclusions.

\section{Problem Formulation and PMSM Model}

Electric vehicles are mainly composed of the electric drive system, battery system, and related auxiliary systems; the simple structure of electric vehicles is shown in Figure 1. The efficiency and safety [17-19] are the key technologies in the battery system. The electric drive system is the core of the energy-consumption component in electric vehicles. For proposal of high-efficiency operation of the battery, highquality motor controller is an essential issue since the electric motor is a high energy-consumption component in the whole electric drive system. Furthermore, the drive motor should have the following metrics: high reliability, highprecision torque, and better dynamic performance to achieve the vehicles' handling stability and driving comfort. However, the existence of external disturbances and parameter perturbation always affects the controller performance of the PMSM system. Therefore, the implementation of the high-performance PMSM control system has become a critical problem to improve the driving range of electric vehicles. Aiming at this issue, a second-order PMSM model with matched and mismatched disturbances is formulated in this section, considering the external disturbances and control accuracy caused by parameter perturbation.

The mathematical model of a surface-mounted PMSM in the $d-q$ frame can be formulated as follows [20]:

$$
\left\{\begin{array}{l}
i_{d}^{\prime}=\frac{u_{d}}{L}-\frac{R_{s}}{L} i_{d}-\omega p_{n} i_{q}, \\
i_{q}^{\prime}=\frac{u_{q}}{L}-\frac{R_{s}}{L} i_{q}-\omega p_{n} i_{d}-\frac{p_{n} \psi_{f}}{L} \omega \\
\dot{\omega}=\frac{3}{2 J} p_{n} i_{q} \psi_{f}-\frac{T_{L}}{J}-\frac{B}{J} \omega
\end{array}\right.
$$

where $i_{d}$ and $i_{q}$ are the $d$-and $q$-axis stator current, $i_{d}^{\prime}$ and $i_{q}^{\prime}$ are the corresponding derivatives, $L$ is the stator inductance, $u_{d}$ and $u_{q}$ are $d$ - and $q$-axis stator voltages, $R_{s}, p_{n}$, and $\omega$ are the stator resistance, number of pole pairs, and angular velocity, respectively, and $\psi_{f}, T_{L}, B$, and $J$ are the flux linkage, bounded load torque, viscous friction coefficient, and moment of inertia.

In the $d-q$ coordinate system, the dynamic equations of the PMSM system are

$$
\dot{\omega}=\frac{3}{2 J_{n}} p_{n} i_{q} \psi_{f n}-\frac{T_{L}}{J_{n}}-\frac{B_{n}}{J_{n}} \omega=b_{n} i_{q}-d-a_{n} \omega,
$$

where $a_{n}=\left(B_{n} / J_{n}\right)$ and $b_{n}=\left(3 p_{n} \psi_{f n} / 2 J_{n}\right)$ are nominal parameters and $d$ is the load disturbance. In addition, $J_{n}, B_{n}$, and $\psi_{f n}$ are the nominal parameters of the PMSM model. For convenience of writing and analysis, we define $x_{1}=\omega_{\text {ref }}-\omega$; the dynamic equation of the PMSM system can be expressed as follows:

$$
x_{2}=\dot{x}_{1}=\dot{\omega}_{\text {ref }}-b_{n} i_{q}+d+a_{n} \omega .
$$

In this paper, considering the effect of the parameter perturbation and external disturbances, parameter uncertainties with the bounds are defined as follows:

$$
\left\{\begin{array}{l}
|\Delta J|=\left|J-J_{n}\right| \leq \bar{\Delta}_{J}, \\
|\Delta B|=\left|B-B_{n}\right| \leq \bar{\Delta}_{B}, \\
\left|\Delta \psi_{f}\right|=\left|\psi_{f}-\psi_{f n}\right| \leq \bar{\Delta}_{\psi_{f n}},
\end{array}\right.
$$




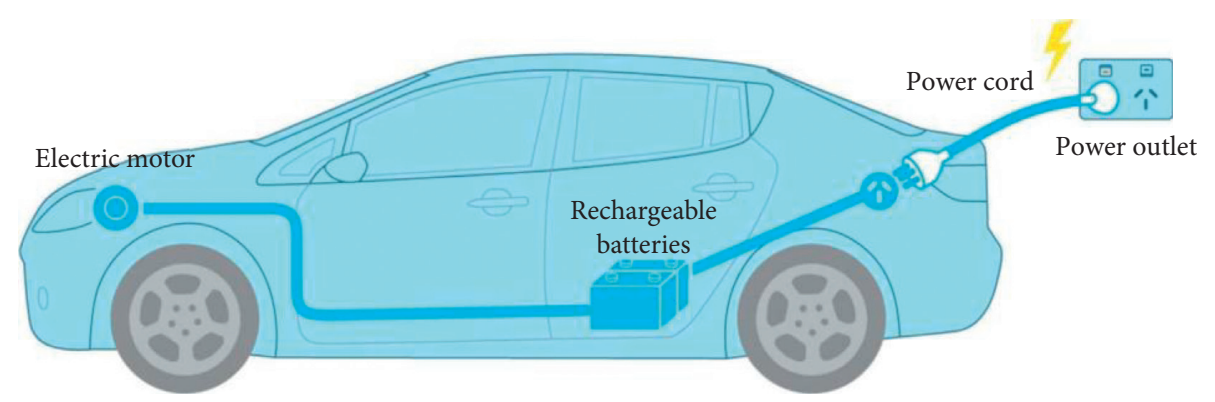

Figure 1: The simple structure of electric vehicles.

where $\bar{\Delta}_{J}, \bar{\Delta}_{B}$, and $\bar{\Delta}_{\psi_{f n}}$ are upper bounds of parameter perturbation. Considering the parameter perturbation and external disturbances, dynamic system (3) is rewritten as

$$
\begin{aligned}
\dot{x}_{1} & =\dot{\omega}_{\text {ref }}-\dot{\omega}=\dot{\omega}_{\text {ref }}-\frac{3\left(\psi_{f n}+\Delta \psi_{f}\right)}{2\left(J_{n}+\Delta J\right)} p_{n} i_{q}+\frac{T_{L}}{\left(J_{n}+\Delta J\right)}+\frac{\left(B_{n}+\Delta B\right)}{\left(J_{n}+\Delta J\right)} \omega, \\
& =x_{2}-\frac{3 p_{n} i_{q}\left(\Delta \varphi_{f n} J_{n}-\varphi_{f n} \Delta J_{n}\right)+2 T_{L} \Delta J_{n}-2 \Delta B_{n} J_{n} \omega+2 B_{n} \Delta J_{n} \omega}{2\left(J_{n}+\Delta J_{n}\right) J_{n}}=x_{2}+d_{1},
\end{aligned}
$$

where $d_{1}$ means the mismatched disturbance that appears in different channels from the control input, which possibly includes load disturbances, unmodeled dynamics, and system disturbance caused by parameter perturbation. Similarly, the dynamic constraints of the system state are obtained as follows:

$$
\begin{aligned}
\dot{x}_{2} & =\ddot{\omega}_{\text {ref }}-\ddot{\omega}=\ddot{\omega}_{\text {ref }}-\frac{3\left(\psi_{f n}+\Delta \psi_{f}\right)}{2\left(J_{n}+\Delta J\right)} p_{n} \dot{i}_{q}+\frac{\dot{T}_{L}}{\left(J_{n}+\Delta J\right)}+\frac{\left(B_{n}+\Delta B\right)}{\left(J_{n}+\Delta J\right)} \dot{\omega} \\
& =-\frac{B_{n}}{J_{n}} x_{2}-\frac{3 p_{n} \psi_{f n}}{2 J_{n}} \dot{i}_{q}+\left[\ddot{\omega}_{\mathrm{ref}}-\frac{3\left(\Delta \psi_{f} J_{n}-\psi_{f n} \Delta J_{n}\right) \dot{i}_{q}+2 J_{n} \dot{T}_{L}+2 J_{n}\left(B_{n}+\Delta B\right) \dot{\omega}_{\mathrm{ref}}-2\left(J_{n} \Delta B-2 B_{n} \Delta J\right) x_{2}}{2\left(J_{n}+\Delta J\right) J_{n}}\right] \\
& =-a_{n} x_{2}-b_{n} u+d_{2}
\end{aligned}
$$

where $d_{2}$ is the disturbance that satisfies the matched condition. The control variable $u=\dot{i}_{q}$ is required to be designed. Therefore, the second-order PMSM model with matched disturbance and mismatched disturbances is given as

$$
\left\{\begin{array}{l}
\dot{x}_{1}=x_{2}+d_{1}, \\
\dot{x}_{2}=-a_{n} x_{2}-b_{n} u+d_{2} .
\end{array}\right.
$$

Note that the above parameter perturbation significantly affects the control performance in reality. Especially, the traditional sliding mode control methods have no ability to handle the appearance of mismatched disturbance. As a result, the PMSM control system should be designed to be unaffected by mismatched disturbance. In addition, the control system should behave with fast response speed and short settling time. The structure of cascade control for the PMSM system is designed in Figure 2. In addition, the reference value of the $d$-axis current is set as zero to implement decouple control. The control target is to solve the speed tracking problem for system (7); meanwhile, eliminating the disturbances impacts on the control system.

Remark 1. As can be seen from (5), the mismatched disturbance involves complex parameter perturbation and the load disturbance. The parameter perturbation mainly results from the moments of inertia, the viscous friction coefficient, and the flux linkage of the PMSM, respectively. In addition, the torque load from the electric vehicles 


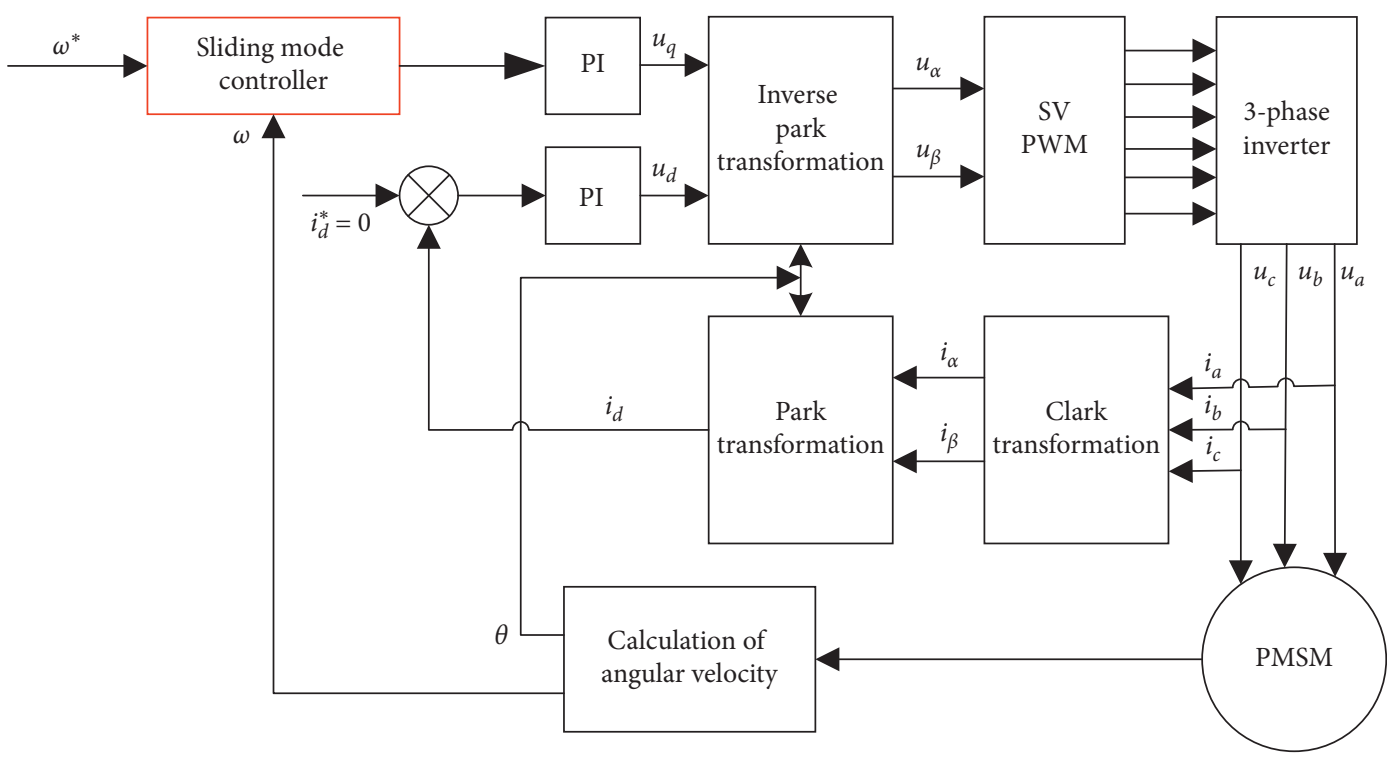

FIgURE 2: The structure of cascade control for the PMSM.

introduces the external load disturbance to the PMSM system. It should be noticed that the above parameter perturbation significantly affects the control performance in reality. On the one hand, mismatched disturbances are always unknown or very difficult to obtain, during the operation of electric vehicles; on the other hand, the traditional sliding mode control methods are insensitive to the mismatched disturbances. Therefore, dealing with the mismatched disturbance and improving control accuracy have been a critical issue in electric vehicles.

\section{Sliding Mode Controller Design Based on the Nonlinear Disturbance Observer}

3.1. Design of the Nonlinear Disturbance Observer. Due to the fact that the mismatched disturbance caused by parameter perturbation always exists in practice, the traditional SMC control methods are insensitive to mismatch the disturbance. Therefore, we first develop a nonlinear disturbance observer (NDO) for estimating matched and mismatched disturbances, which is the foundation of controller design. According to the system model in (7), the NDO is designed as

$$
\left\{\begin{array}{l}
\dot{\hat{x}}_{1}=x_{2}+z_{1}, \\
z_{1}=-\lambda_{1} \operatorname{sig}^{(2 / 3)}\left(\hat{x}_{1}-x_{1}\right)+\widehat{d}_{1} \\
\dot{\hat{d}}_{1}=-\lambda_{2} \operatorname{sig}^{(1 / 2)}\left(\hat{d}_{1}-z_{1}\right) \\
\dot{\hat{x}}_{2}=-a_{n} x_{2}-b_{n} u+z_{2} \\
z_{2}=-\lambda_{3} \operatorname{sig}^{(2 / 3)}\left(\hat{x}_{2}-x_{2}\right)+\widehat{d}_{2} \\
\dot{\vec{d}}_{2}=-\lambda_{4} \operatorname{sig}^{(1 / 2)}\left(\hat{d}_{2}-z_{2}\right)
\end{array}\right.
$$

where $\widehat{x}_{1}$ and $\widehat{x}_{2}$ are the velocity error and its deviation; $\widehat{d}_{1}$ and $\widehat{d}_{2}$ are the estimation value of the disturbance; $z_{1}$ and $z_{2}$ are intermediate variables; and $\lambda_{1}, \lambda_{2}, \lambda_{3}$, and $\lambda_{4}$ are strictly positive gains of the nonlinear disturbance observer.

Taking the observer errors as $e_{x_{1}}=x_{1}-\widehat{x}_{1}, e_{x_{2}}=x_{2}-\widehat{x}_{2}$, $e_{d_{1}}=d_{1}-\widehat{d}_{1}$, and $e_{d_{2}}=d_{2}-\widehat{d}_{2}$, the observer equations are formulated as

$$
\left\{\begin{array}{l}
\dot{e}_{x_{1}}=-\lambda_{1} \operatorname{sig}^{(2 / 3)}\left(e_{x_{1}}\right)+e_{d_{1}}, \\
\dot{e}_{d_{1}}=-\lambda_{2} \operatorname{sig}^{(1 / 2)}\left(e_{d_{1}}-\dot{e}_{x_{1}}\right)+\dot{d}_{1} \\
\dot{e}_{x_{2}}=-\lambda_{3} \operatorname{sig}^{(2 / 3)}\left(e_{x_{2}}\right)+e_{d_{2}}, \\
\dot{e}_{d_{2}}=-\lambda_{4} \operatorname{sig}^{(1 / 2)}\left(e_{d_{2}}-\dot{e}_{x_{2}}\right)+\dot{d}_{2} .
\end{array}\right.
$$

It follows from the proof [21] that all the estimation terms and their derivations are always bounded, and positive gains $\lambda_{1}, \lambda_{2}, \lambda_{3}$, and $\lambda_{4}$ exist to drive the observer to have a finite-time convergence performance. In other words, there is a sample time $T^{*}$, and $e_{x_{1}}, e_{d_{1}}, e_{x_{2}}$, and $e_{d_{2}}$ are bounded, when $t \in\left(0, T^{*}\right]$. When $t>T^{*}$, the observer error can be regarded as $e_{x_{1}}=0, e_{d_{1}}=0, e_{x_{2}}=0$, and $e_{d_{2}}=0$.

Remark 2. The parameters of the nonlinear disturbance observer should be carefully chosen in practical implementations. Generally, large values of $\lambda_{1}, \lambda_{2}, \lambda_{3}$, and $\lambda_{4}$ can improve the convergence performance of the nonlinear disturbance observer, but too large ones will induce severe chattering of the sliding surface, and it further weakens the tracking performance of the PMSM system. As a result, we should carefully study the mismatching disturbance caused by uncertainty factors and their amplitudes in the operation of electric vehicles. In such cases, the NDO parameters can be selected properly in practical implementation.

3.2. Design of the Novel Sliding Mode Controller. In order to effectively suppress the mismatched disturbance in system (7) and improve the robustness of the PMSM system, an observer value of the NDO is introduced into the sliding 
surface. Therefore, a PID-type sliding surface with the disturbance observer is designed:

$$
s=c_{1} x_{1}+\left(\widehat{d}_{1}+x_{2}\right)+c_{2} \int_{0}^{t} x_{1} \mathrm{~d} \tau
$$

where $c_{1}$ and $c_{2}$ are positive gains and $\widehat{d}_{1}$ is the estimation of the mismatched disturbance.

Remark 3. Generally, large gains of $c_{1}$ and $c_{2}$ can improve the response speed and accuracy, but too large ones will induce large chattering and control overshoot. For the practical applications, we need to carefully investigate the characteristic of the closed-loop system and consider the tradeoff between the response speed and the overshoot.

According to Lyapunov stability theory, to ensure system stability and performance, a new sliding mode control law based on the mismatched disturbance observer is designed as

$$
u=\frac{1}{b_{n}}\left[\left(c_{1}-a_{n}\right) x_{2}+c_{2} x_{1}+\widehat{d}_{2}+c_{1} \widehat{d}_{1}+k \operatorname{sgn}(s)+q s\right],
$$

where $k$ is the switching gain to be designed and $q$ is the designed parameter in the reaching law. The detailed control block on implementation of the presented NDO-SMC is shown in Figure 3.

Assumption 1. The mismatched disturbance $d_{1}$ and its deviation are bounded. Thus. we have

$$
\left|\frac{\mathrm{d}^{j} \mathrm{~d}_{1}}{\mathrm{~d} t^{j}}\right| \leq \mu, \quad j=0,1,2, \ldots, r,
$$

where $\mu$ is a positive number. It is worth mentioning that we just need to know the boundedness of disturbance, rather than the bound value $\mu$. In addition, this assumption is reasonable. From the above analysis, we can see that the influence of parameter perturbation and load disturbance are both limited, which will inevitably lead to the boundedness of mismatched disturbance.

Theorem 1. Suppose that Assumption 1 holds. Considering system (7) under the presented control law (11), the closedloop system is stable, if the switching gain satisfies $k>\delta_{0}$, on the premise of finite-time convergence of the nonlinear disturbance observer, where $\delta_{0}=\max \left\{\mu+e_{d_{2}}^{*}+c_{1} e_{d_{1}}^{*}\right\}$, $e_{d_{1}}^{*}=\sup _{t \in}\left[0, T^{*}\right]\left\{e_{d_{1}}\right\}$, and $e_{d_{2}}^{*}=\sup _{t \in\left[0, T^{*}\right]}\left\{e_{d_{2}}\right\}$.

Proof. Taking the derivative of sliding surface $s$ defined in (10) yields

$$
\dot{s}=c_{1}\left(x_{2}+d_{1}\right)+\dot{\hat{d}}_{1}+\dot{x}_{2}+c_{2} x_{1} \text {. }
$$

Substituting the control law (11) into (13) gives

$$
\dot{s}=\dot{\hat{d}}_{1}+\left(d_{2}-\widehat{d}_{2}\right)+c_{1}\left(d_{1}-\widehat{d}_{1}\right)-k \operatorname{sgn}(s)-q s .
$$

Considering the boundedness of matched and mismatched disturbances, formula (14) can be rewritten as

$$
\dot{s}=\dot{\vec{d}}_{1}+e_{d_{2}}+c_{1} e_{d_{1}}-k \operatorname{sgn}(s)-q s .
$$

Consider a candidate Lyapunov function as follows:

$$
V=0.5 s^{2}
$$

Taking the derivative of Lyapunov function $V$ in (16) gives

$$
\begin{aligned}
\dot{V} & =s \dot{s}, \\
& =s\left[\dot{\hat{d}}_{1}+e_{d_{2}}+c_{1} e_{d_{1}}-k \operatorname{sgn}(s)-q s\right], \\
& \leq s\left[\mu+e_{d_{2}}^{*}+c_{1} e_{d_{1}}^{*}-k \operatorname{sgn}(s)-q s\right], \\
& \leq-\left(k-\delta_{0}\right)|s|
\end{aligned}
$$

where $\delta_{0}=\max \left\{\mu+e_{d_{2}}^{*}+c_{1} e_{d_{1}}^{*}\right\}, e_{d_{1}}^{*}=\sup _{t \in\left[0, T^{*}\right]}\left\{e_{d_{1}}\right\}$, and $e_{d_{2}}^{*}=\sup _{t \in\left[0, T^{*}\right]}\left\{e_{d_{2}}\right\}$. According to the finite-time observer theory, the boundedness of the observer error must lead to the existence of $\delta_{0}$. It demonstrates that $\dot{V}<0$ is satisfied, when $k>\delta_{0}$. In such cases, the existence and reachability of sliding modes are satisfied, which ensures that the sliding modes are invariant to mismatched and matched disturbances, and the robustness of the whole system is proved. Therefore, the proposed NDO-SMC strategy has strong robustness against disturbances.

\section{Simulation and Results' Analysis}

To verify the effectiveness of the proposed NDO-SMC strategy, comprehensive simulations have been conducted in this section, and the main parameters of the PMSM are summarized in Table 1. It should be mentioned that the sample frequency of simulation is set as $10^{5}$ to guarantee fair comparison. To illustrate the superiority and effectiveness of the proposed controller, the simulations are conducted from two aspects: (1) external disturbance rejection ability; (2) robustness against model uncertainties; (3) the superiority validation compared with ISMC method. The simulations are shown as follows.

4.1. External Disturbance Rejection Ability. During the operation of electric vehicles, the PMSM is easy to be affected by external load disturbances. Therefore, the ability to suppress external disturbances becomes an essential performance criterion. In this section, the external disturbance rejection ability of the PMSM system under the NDO-SMC scheme is investigated, and the controller parameters are shown in Table 2. Considering the case that the external load disturbance $T_{L}=6(\mathrm{~N} / \mathrm{m})$ is imposed on systems at $t=0.2 \mathrm{~s}$, the corresponding response curves of the PMSM system are shown in Figure 4.

Figure 4(a) shows the speed response with SMC and NDO-SMC strategies. Brief observation (there is no external disturbance before $0.2 \mathrm{~s}$ ) shows that the proposed method results in better responses as those of the baseline SMC method, which verifies the nominal performance recovery of the proposed methods. In the case of external load disturbances, the SMC strategy without disturbance compensation 


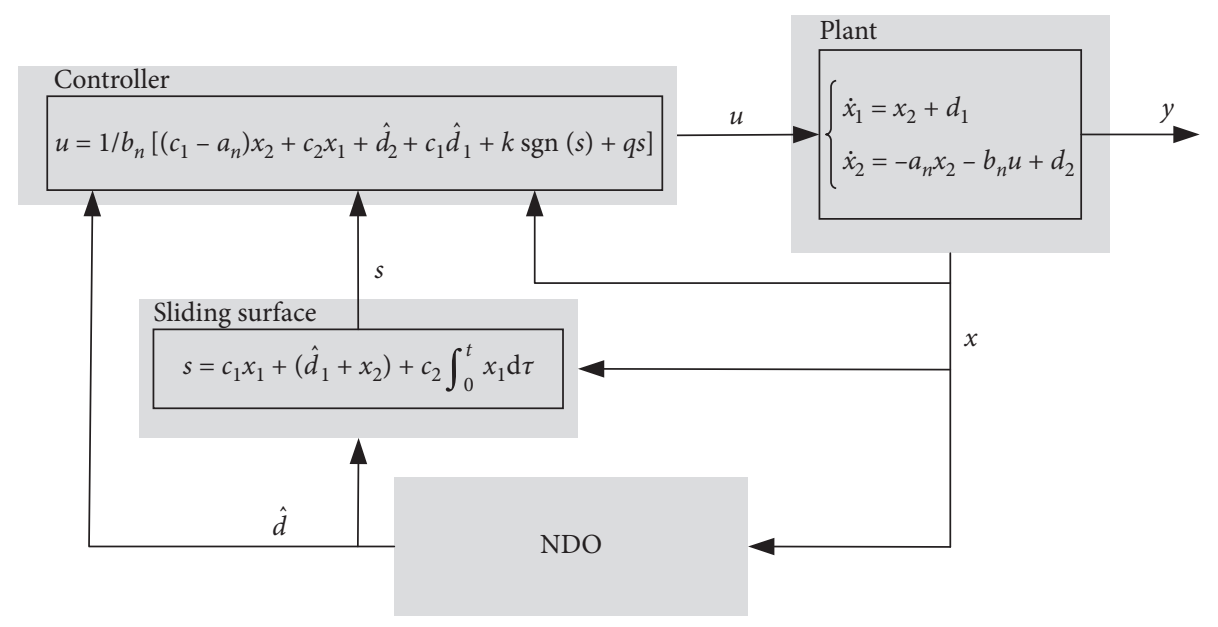

FIgURE 3: The control block of the NDO-SMC scheme.

TABLE 1: The main parameters of the PMSM.

\begin{tabular}{lccc}
\hline Rated power & $3 \mathrm{~kW}$ & Stator inductance & $8.5 \mathrm{mH}$ \\
Rated current & $5.4 \mathrm{~A}$ & Viscous coefficient & $0.008 \mathrm{Nms} / \mathrm{rad}$ \\
Flux linkage & $0.175 \mathrm{~Wb}$ & Pole pairs & 4 \\
Rotor inertia & $0.003 \mathrm{kgm}^{2}$ & Stator resistance & $2.875 \Omega$ \\
\hline
\end{tabular}

TABle 2: The parameters of the controller scheme.

\begin{tabular}{cccc}
\hline$\lambda_{1}$ & 50 & $c_{1}$ & 30 \\
$\lambda_{2}$ & 8000 & $c_{2}$ & 0.5 \\
$\lambda_{3}$ & 100 & $k$ & 20000 \\
$\lambda_{4}$ & 11800 & $q$ & 300 \\
\hline
\end{tabular}

results in large speed fluctuation and overshoot; it further leads to longer settle time of the PMSM system. While the NDO-SMC strategy obtains fine disturbance rejection property, the control system has a much better transient and dynamic performance, such as small overshoot and short settling time. Especially, the switching gain of the controller is substantially reduced due to the compensation of the nonlinear disturbance observer; it also leads to the reduction of system chattering, which is shown in Figure 4(b). The phase trajectory of two methods is shown in Figure 5. It can be seen that two control schemes can converge to the origin, which is consistent with our analysis. In addtion, the chattering of the phase trajectory in the NDO-SMC method is caused by the addition of mismatched disturbance. Therefore, compared with the SMC strategy, NDO-SMC can effectively suppress the external load disturbance, and it also has strong robustness and excellent dynamic performance.

4.2. Robustness against Model Uncertainties. As we all know, with the use of electric vehicles, the nominal parameters of motors will change. Therefore, in order to validate the influence of model parameter perturbation on the accuracy of the PMSM system, we set the model parameters as $90 \%$ of nominal parameters in the system, i.e., $J=0.9 J_{n}$. In addition, the controller parameters are set as the nominal parameters of the PMSM. The reference speed changes from
$500 \mathrm{r} / \mathrm{min}$ to $550 \mathrm{r} / \mathrm{min}$. The simulation results of the controller are shown in Figure 6.

It can be seen from Figure 6 that the NDO-SMC scheme can improve the convergence speed and effectively reduce the overshoot of the system in the start-up phase due to the compensation of the NDO. In addition, when the reference speed of the system increases, the response time of the SMC control scheme increases obviously, and the system overshoot is larger, and it obviously cannot meet the control accuracy of electric vehicles in complex driving cycles, while the proposed NDO-SMC scheme can provide higher torque for improving the dynamic response of the system, such as short setting time and small overshoot.

In addition, in order to verify the effectiveness of the algorithm under different degrees of parameter perturbation, using the same scheme, we define the amplitude of parameter perturbation as $70 \%, 80 \%, 90 \%, 100 \%, 110 \%$, and $120 \%$ of nominal parameter $J_{n}$, while keeping the controller parameters unchanged.

Figures 7(a) and 7(b) show the control effect under the SMC method. Figures $7(\mathrm{c})$ and $7(\mathrm{~d})$ show the control performance under the NDO-SMC scheme. It can be seen from the figures that different degrees of parameter perturbation have different impacts on the system response under the SMC scheme. The smaller the parameter perturbation range is, the smaller the influence on the control performance will be. When the parameter perturbation range reaches a certain boundary value of $70 \%$, it will cause drastic changes in the system and even lead to the instability of the control system. However, under the NDO-SMC scheme, the impact on the system response caused by different range of parameter perturbation will be suppressed or even be eliminated. When the parameter perturbation range is large, the system response will be slightly different. The simulation results show that the proposed NDO-SMC method has strong robustness against model uncertainties caused by parameter perturbation of the system model. This scheme can effectively reduce the influence of parameter perturbation in the 


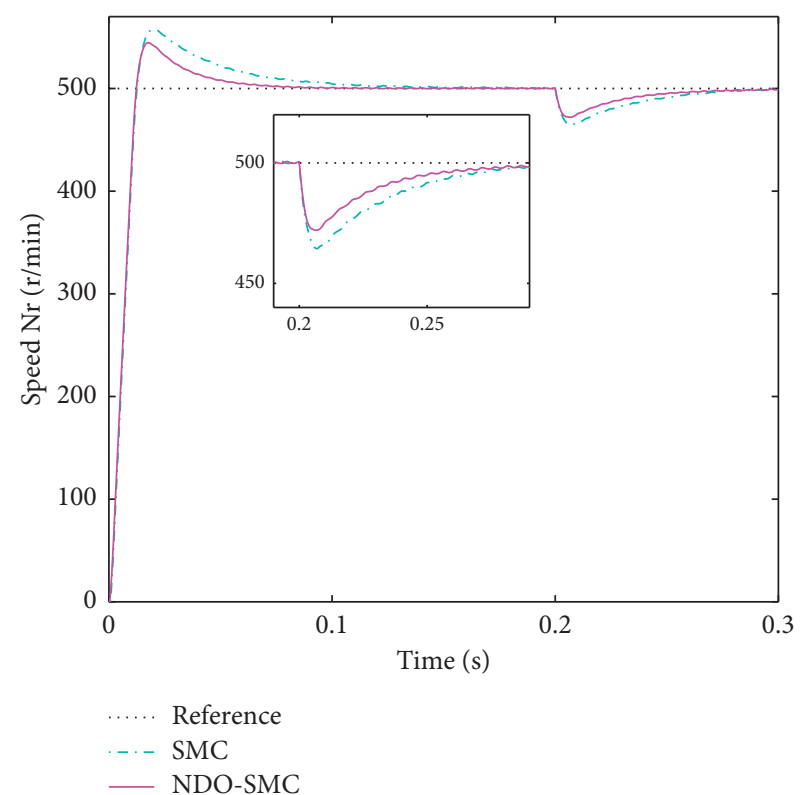

(a)

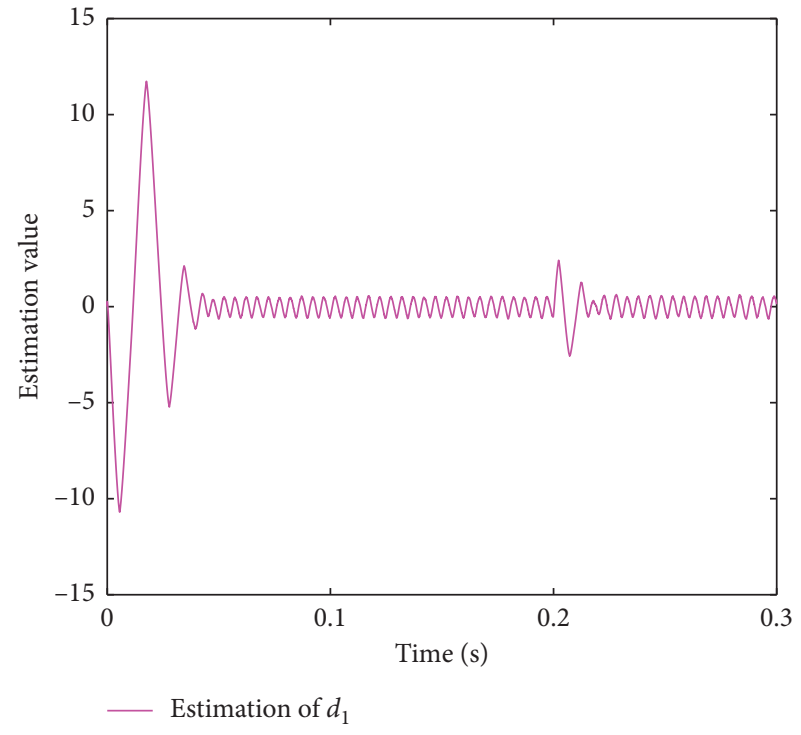

(c)

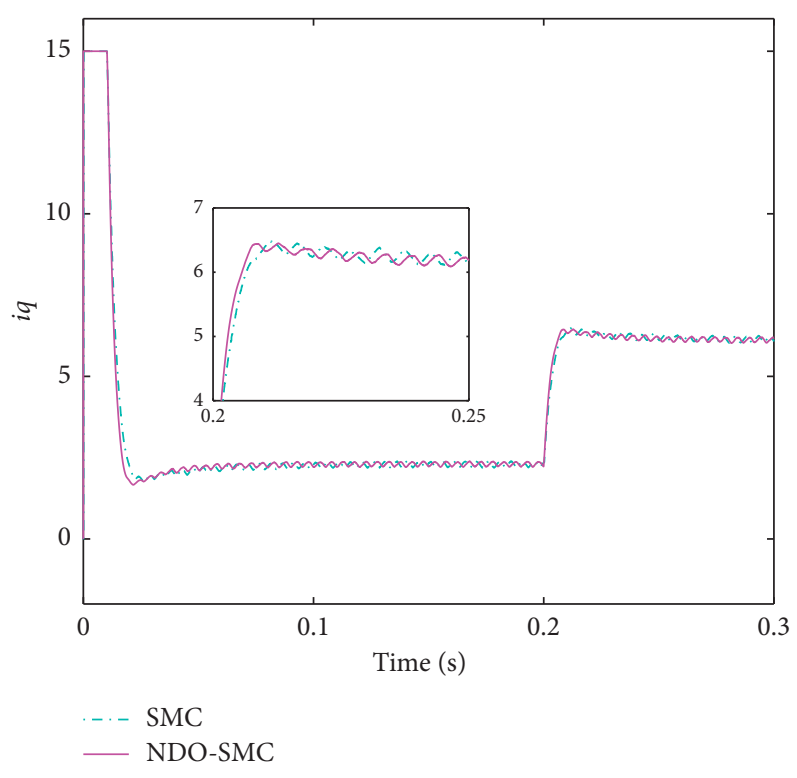

(b)

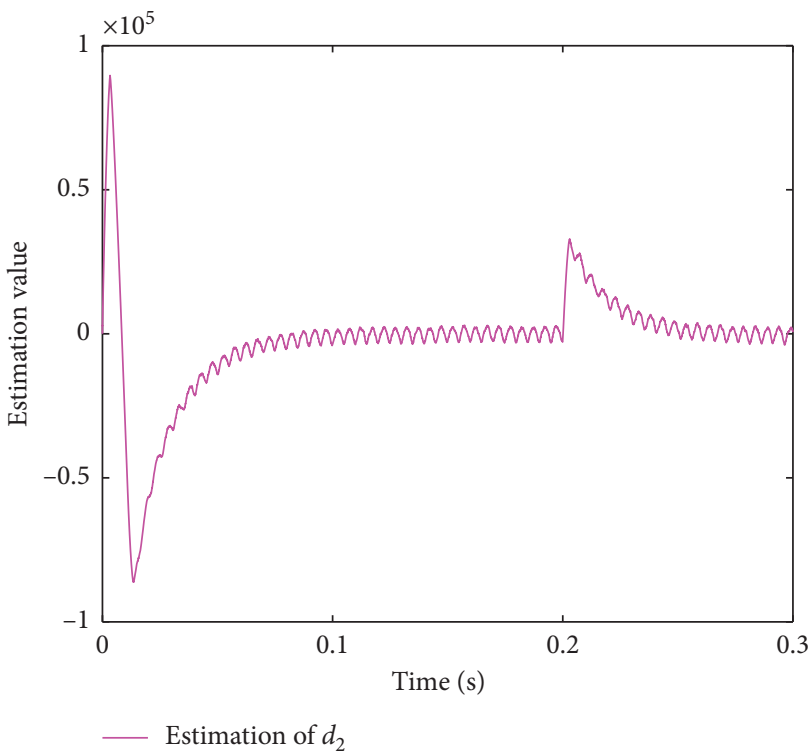

(d)

FIgURE 4: The validation of the external disturbance rejection ability. (a) Speed curve. (b) Response curve of $q$-axis current. (c) Estimation of $d_{1}$. (d) Estimation of $d_{2}$.

actual operation of electric vehicles and improve the robustness of motor control.

In order to intuitively exhibit the performance of the NDO-SMC method, overshoot and settling time (3\% steadystate error) of the PMSM system are used to demonstrate. The simulation results are shown in Table 3.

As can be seen from Table 3, when the system has the same range of parameter perturbation, the overshoot and settling time of the proposed NDO-SMC scheme are significantly lower than those of the SMC method. In addition, when the parameter perturbation range is greater than $30 \%$, the settling time of the SMC scheme can no longer meet the requirements of the real-time control system, while the NDO-SMC scheme can also meet the basic requirements of the PMSM system.

4.3. Superiority of the NDO-SMC Scheme. For the purpose of superiority validation, the NDO-SMC scheme and ISMC [14] are compared. In this section, we set the model parameters as $90 \%$ of nominal parameters in the system, i.e., $J=0.9 J_{n}$. In addition, the controller parameters are set as 


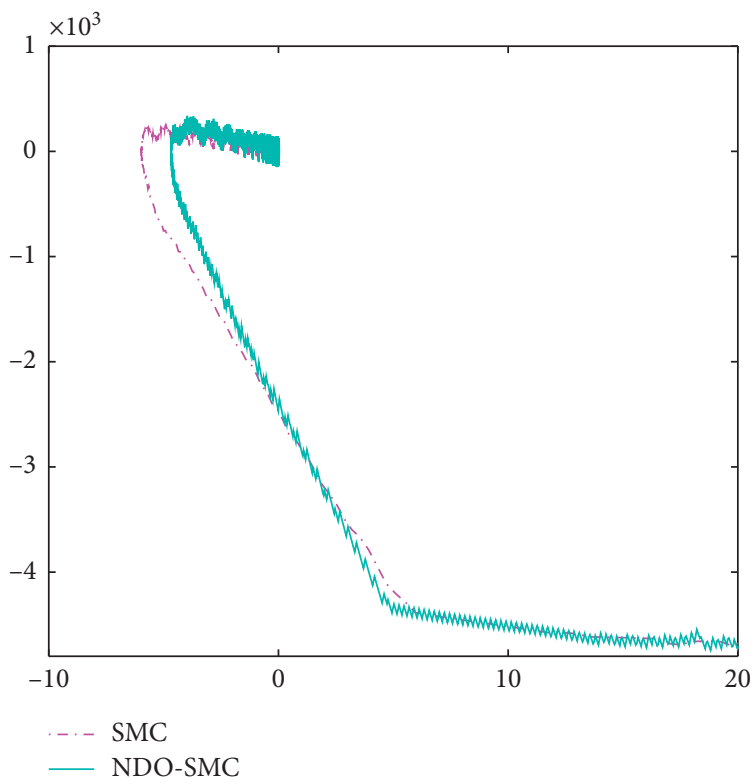

Figure 5: The phase trajectories of SMC and NDO-SMC methods.

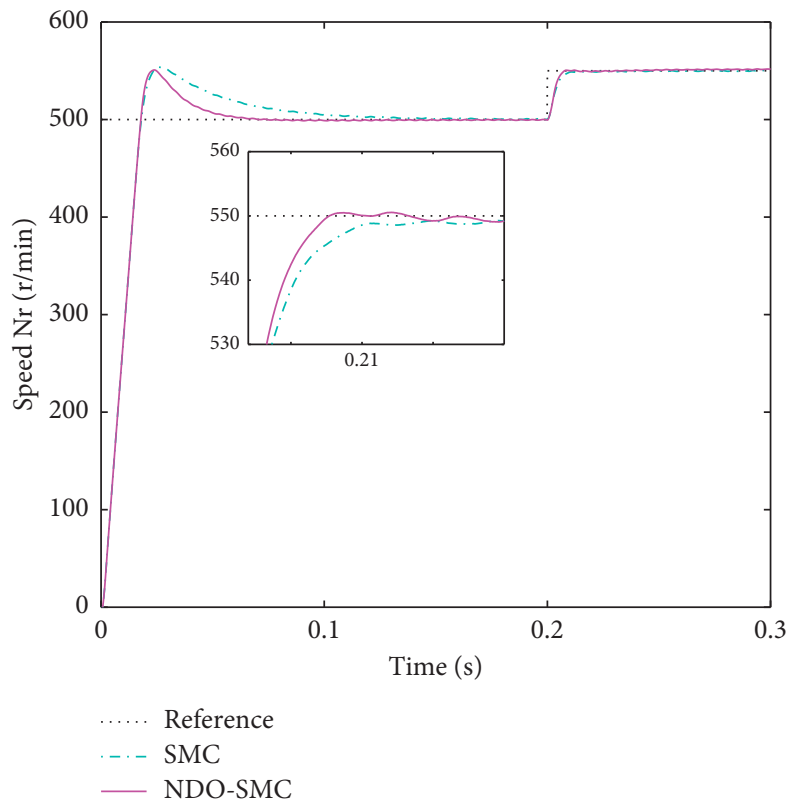

(a)

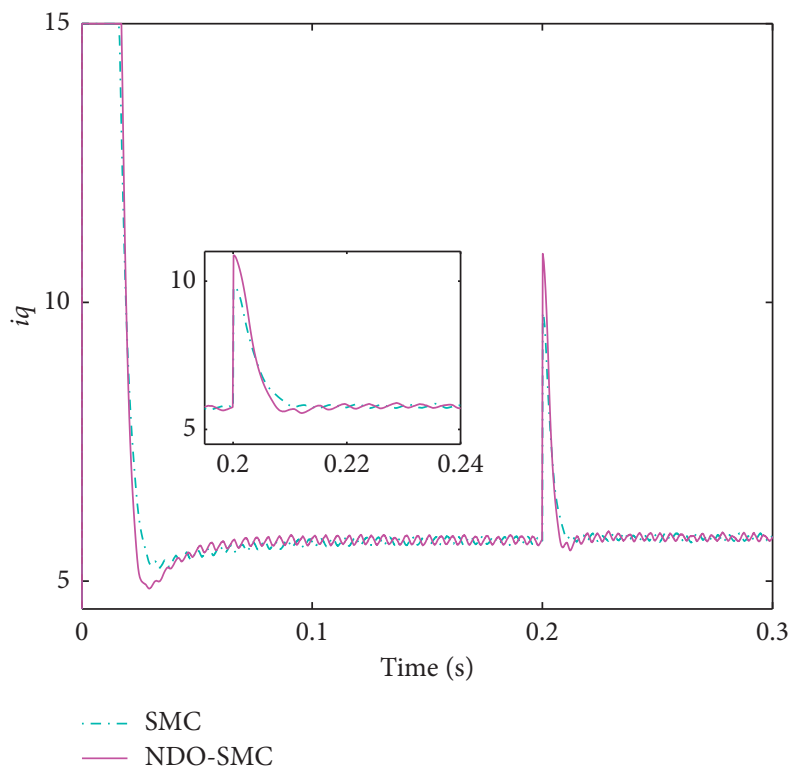

(b)

FIgURE 6: The validation of robustness against parameter perturbation $\left(J=0.9 J_{n}\right)$. (a) Speed curve. (b) Response curve of $q$-axis current.

the nominal parameters of the PMSM. The reference speed increases from $500 \mathrm{r} / \mathrm{min}$ to $550 \mathrm{r} / \mathrm{min}$ at $t=0.2 \mathrm{~s}$. The comparison results of the two controllers are shown in Figure 8.

In general, the proposed NDO-SMC scheme has the characteristics of integral term, where the sliding surface is defined as $s=c_{1} x_{1}+\left(\widehat{d}_{1}+x_{2}\right)+c_{2} \int_{0}^{t} x_{1} \mathrm{~d} \tau$. The NDO-SMC method eliminates the mismatched disturbance by adding the estimated value $\widehat{d}_{1}$ of the mismatched disturbance to the sliding variable $s$. In this way, the effect of mismatched disturbance can be eliminated, once mismatched disturbance can be estimated accurately, while ISMC mainly relies on the integral action to drive the system state to approach the origin and eliminate the mismatched disturbance. However, integral action will cause serious overshoot of the system, which will affect the dynamic performance of the 

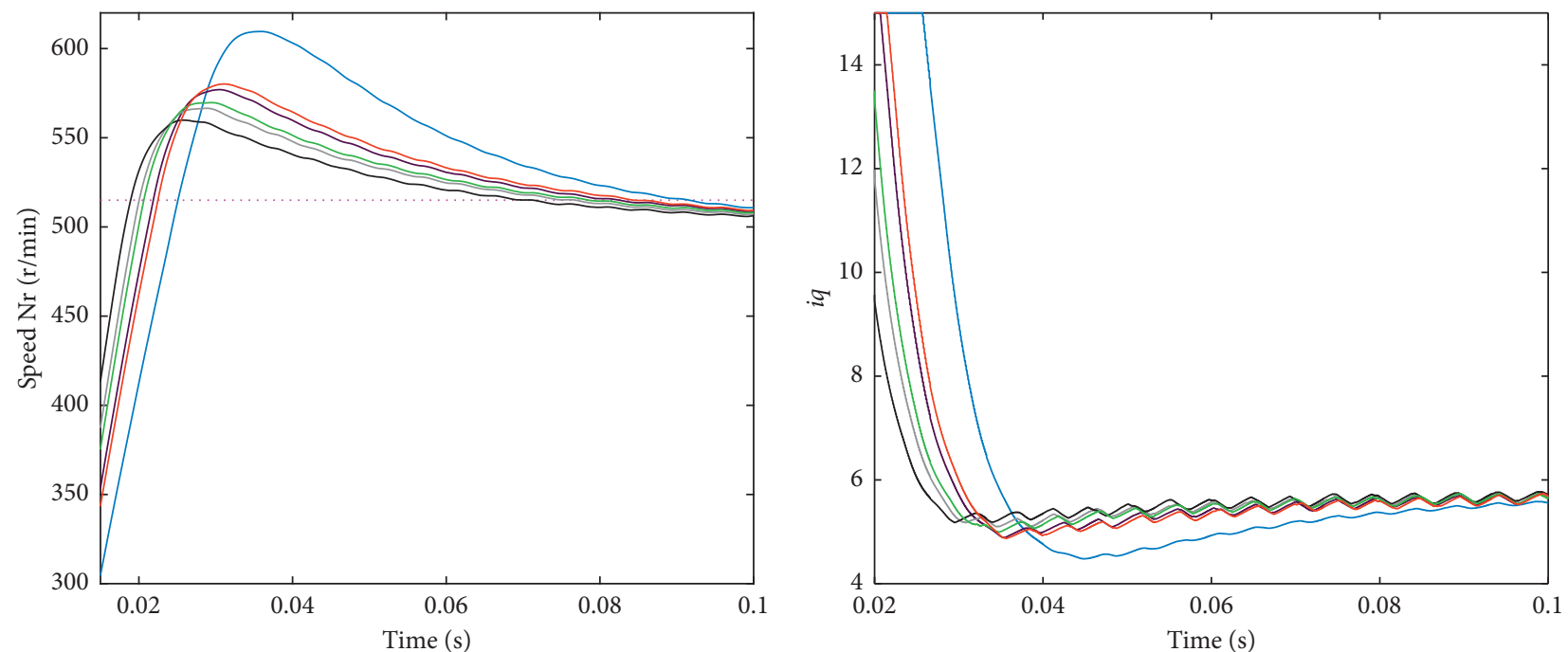

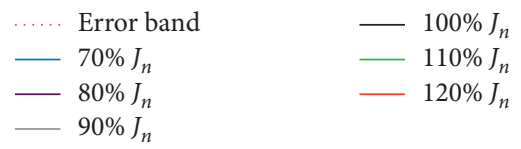

(a)

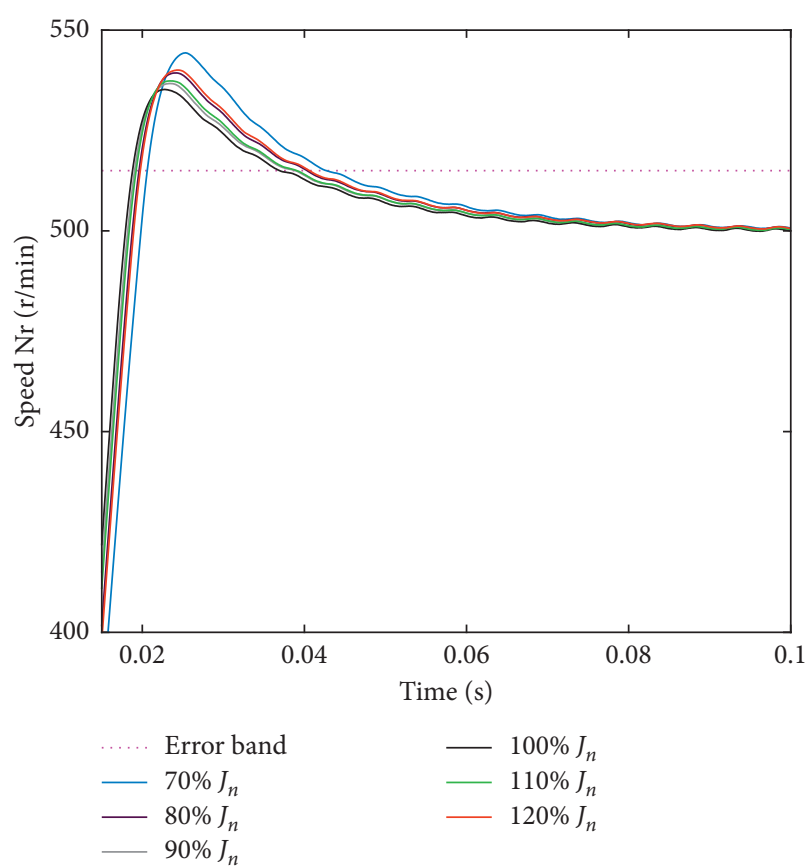

(c)

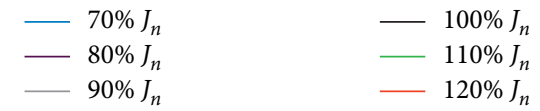

(b)

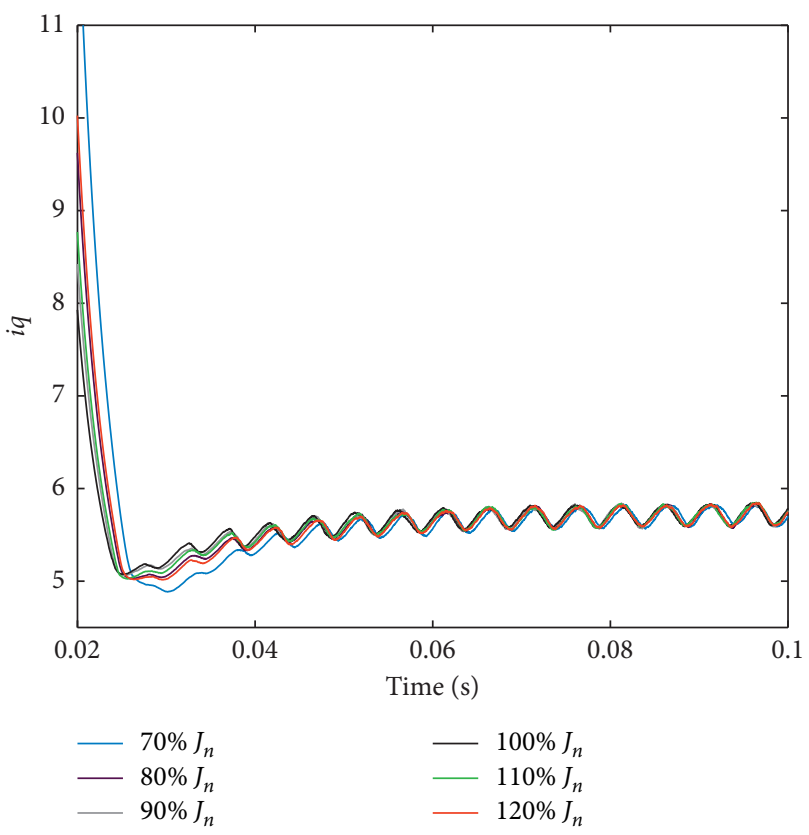

(d)

FIGURE 7: The robustness validation under different amplitudes of parameter perturbation. (a) Speed curve under the SMC scheme. (b) $q$-axis current curve under the SMC scheme. (c) Speed curve under the NDO-SMC scheme. (d) $q$-axis current curve under the NDO-SMC scheme. 
TABLE 3: The performance index of two schemes.

\begin{tabular}{lcccc}
\hline & \multicolumn{2}{c}{ SMC scheme (speed) } & \multicolumn{2}{c}{ NDO-SMC scheme (speed) } \\
& Overshoot $(\%)$ & Settling time $(\mathrm{s})$ & Overshoot $(\%)$ & 0.0427 \\
\hline $70 \% J_{n}$ & 21.91 & 0.094 & 8.87 & 0.0403 \\
$80 \% J_{n}$ & 15.40 & 0.083 & 7.87 & 0.0387 \\
$90 \% J_{n}$ & 13.30 & 0.077 & 7.05 & 0.037 \\
$100 \% J_{n}$ & 11.97 & 0.072 & 7.47 & 0.0391 \\
$110 \% J_{n}$ & 13.74 & 0.079 & 8.01 & 0.0406 \\
$120 \% J_{n}$ & 16.03 & 0.085 & & Settling time \\
\hline
\end{tabular}

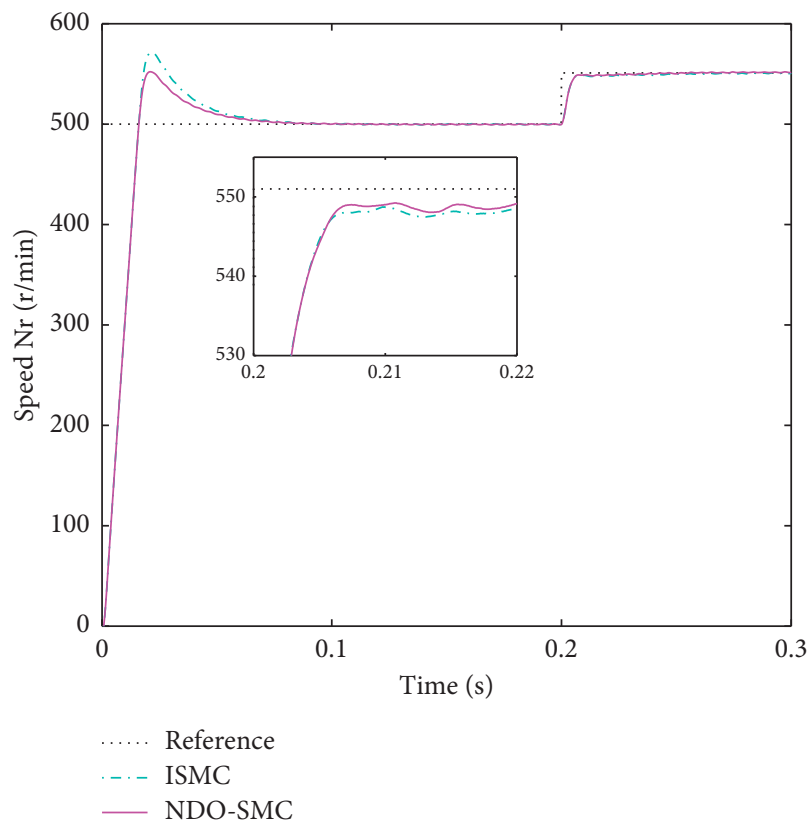

(a)

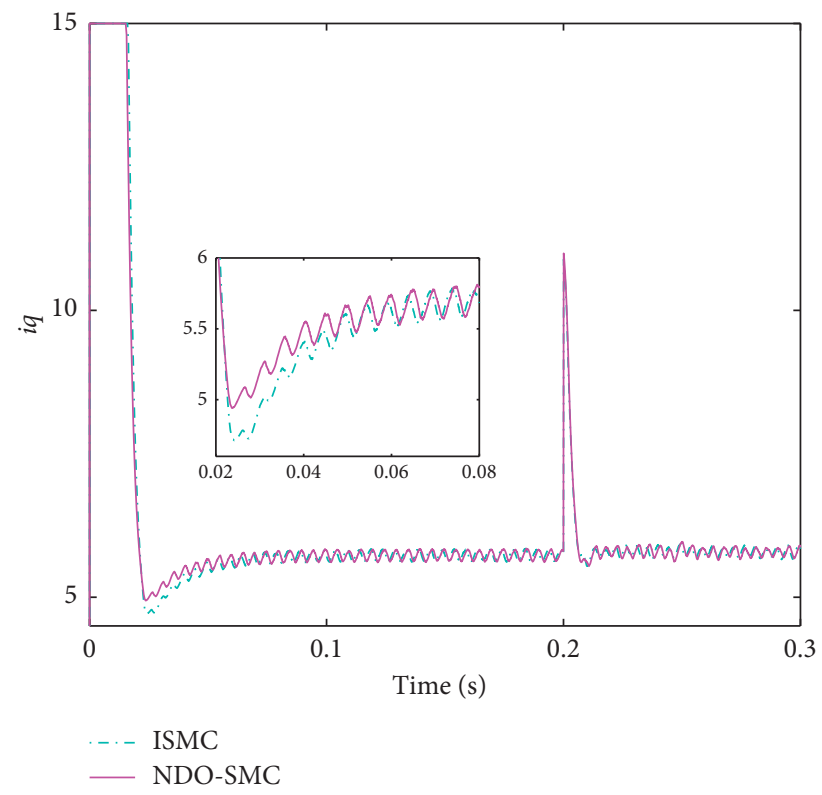

(b)

Figure 8: The simulation results compared with the ISMC method $\left(J=0.9 J_{n}\right)$. (a) Speed curve. (b) Response curve of $q$-axis current.

system. As is shown in Figure 8, the proposed NDO-SMC approach can suppress overshoot and improve the dynamic performance of the PMSM system, which is consistent with the above analysis. In summary, the proposed scheme provides a simple and effective way to deal with the mismatched disturbance.

\section{Conclusion}

This paper has proposed a NDO-SMC scheme for PMSM systems with matched and mismatched disturbances, where the observer estimation is involved in the sliding surface to drive the system state to the desired equilibrium point in the presence of mismatched disturbances. The main contribution is to develop a new high-performance scheme for the PMSM to attenuate disturbances and improve the efficiency of batteries, considering the disturbance problem in the operation of electric vehicles. The proposed controller not only exhibits an excellent robustness performance against the external disturbance and parameter perturbation but also reveals chattering reduction by the compensation of the nonlinear disturbance observer. A series of simulations are conducted to demonstrate the feasibility as well as superiority of the proposed scheme. The simulation results have revealed that the proposed scheme has the properties of chattering reduction as well as excellent dynamic performance, compared with the SMC method.

\section{Data Availability}

The data used to support the findings of this study are available from the corresponding author upon request.

\section{Disclosure}

The authors declare that the work described was original research that has not been published previously and not under consideration for publication elsewhere, in whole or in part.

\section{Conflicts of Interest}

The authors declare no conflicts of interest. 


\section{Authors' Contributions}

All the authors approved the manuscript that is enclosed.

\section{Acknowledgments}

This work was supported by the National Science and Technology Support Program (Grant no. 2014BAG06B02) and Fundamental Research Funds for the Central Universities (Grant no. 2014HGCH0003).

\section{References}

[1] E. A. Grunditz, T. Thiringer, and N. Saadat, "Acceleration, drive cycle efficiency, and cost tradeoffs for scaled electric vehicle drive system," IEEE Transactions on Industry Applications, vol. 56, no. 3, pp. 3020-3033, 2020.

[2] C. Zhang, Y. He, L. Yuan, and S. Xiang, "Capacity prognostics of lithium-ion batteries using EMD denoising and multiple Kernel RVM," IEEE Access, vol. 5, pp. 12061-12070, 2017.

[3] X. Liu, H. Chen, J. Zhao, and A. Belahcen, "Research on the performances and parameters of interior PMSM used for electric vehicles," IEEE Transactions on Industrial Electronics, vol. 63, no. 6, pp. 3533-3545, 2016.

[4] Y. Wang, Y. Feng, X. Zhang, and J. Liang, "A new reaching law for antidisturbance sliding-mode control of PMSM speed regulation system," IEEE Transactions on Power Electronics, vol. 35, no. 4, pp. 4117-4126, 2020.

[5] J. Li, H.-P. Ren, and Y.-R. Zhong, "Robust speed control of induction motor drives using first-order auto-disturbance rejection controllers," IEEE Transactions on Industry Applications, vol. 51, no. 1, pp. 712-720, 2015.

[6] M. Preindl and S. Bolognani, "Model predictive direct torque control with finite control set for PMSM drive systems, part 1 : maximum torque per ampere operation," IEEE Transactions on Industrial Informatics, vol. 9, no. 4, pp. 1912-1921, 2013.

[7] F.-J. Lin, I.-F. Sun, J.-K. Yang, and J. K. Chang, "Intelligent position control of permanent magnet synchronous motor using recurrent fuzzy neural cerebellar model articulation network," IET Electric Power Applications, vol. 9, no. 3, pp. 248-264, 2015.

[8] Y. T. Deng, J. L. Wang, H. W. Li, J. Liu, and D. P. Tian, "Adaptive sliding mode current control with sliding mode disturbance observer for PMSM drives," ISA Transactions, vol. 82, pp. 113-126, 2018.

[9] H. Yan, X. Zhou, H. Zhang, F. Yang, and Z.-G. Wu, “A novel sliding mode estimation for microgrid control with communication time delays," IEEE Transactions on Smart Grid, vol. 10, no. 2, pp. 1509-1520, 2019.

[10] C. J. Kempf and S. Kobayashi, "Disturbance observer and feedforward design for a high-speed direct-drive positioning table," IEEE Transactions on Control Systems Technology, vol. 7, no. 5, pp. 513-526, 1999.

[11] J. Yang, W.-H. Chen, and S. Li, "Non-linear disturbance observer-based robust control for systems with mismatched disturbances/uncertainties," IET Control Theory \& Applications, vol. 5, no. 18, pp. 2053-2062, 2011.

[12] J. Yang, W.-H. Chen, S. Li, L. Guo, and Y. Yan, "Disturbance/ uncertainty estimation and attenuation techniques in PMSM drives-a survey," IEEE Transactions on Industrial Electronics, vol. 64, no. 4, pp. 3273-3285, 2017.

[13] D. Ginoya, P. D. Shendge, and S. B. Phadke, "Sliding mode control for mismatched uncertain systems using an extended disturbance observer," IEEE Transactions on Industrial Electronics, vol. 61, no. 4, pp. 1983-1992, 2014.

[14] L. Zhou, Z. Che, and C. Yang, "Disturbance observer-based integral sliding mode control for singularly perturbed systems with mismatched disturbances," IEEE Access, vol. 6, pp. 9854-9861, 2018.

[15] J. Yang, J. Su, S. Li, and X. Yu, "High-order mismatched disturbance compensation for motion control systems via a continuous dynamic sliding-mode approach," IEEE Transactions on Industrial Informatics, vol. 10, no. 1, pp. 604-614, 2014.

[16] J. Yang, S. Li, and X. Yu, "Sliding-mode control for systems with mismatched uncertainties via a disturbance observer," IEEE Transactions on Industrial Electronics, vol. 60, no. 1, pp. 160-169, 2013.

[17] C. Zhang, Y. He, B. Du, L. Yuan, B. Li, and S. Jiang, "Transformer fault diagnosis method using iot based monitoring system and ensemble machine learning," Future Generation Computer Systems, vol. 108, pp. 533-545, 2020.

[18] C. Zhang, Y. He, S. Jiang, T. Wang, L. Yuan, and B. Li, "Transformer fault diagnosis method based on self-powered RFID sensor tag, DBN, and MKSVM," IEEE Sensors Journal, vol. 19, no. 18, pp. 8202-8214, 2019.

[19] C. Zhang, Y. He, L. Yuan, and S. Xiang, "Analog circuit incipient fault diagnosis method using dbn based features extraction," IEEE Access, vol. 6, pp. 23053-23064, 2018.

[20] P. Pei, Z. Pei, Z. Tang, and H. Gu, "Position tracking control of PMSM based on fuzzy PID-variable structure adaptive control," Mathematical Problems in Engineering, vol. 2018, Article ID 5794067, 15 pages, 2018.

[21] A. Levant, "Finite differences in homogeneous discontinuous control," IEEE Transactions on Automatic Control, vol. 52, no. 7, pp. 1208-1217, 2007. 